BMJ Open

Sport \&

Exercise

Medicine

\title{
Descriptive study of female roller derby athletes' beliefs about risk factors for injury in roller derby
}

\author{
Michelle L Cathorall, Megan Punches
}

To cite: Cathorall ML, Punches M. Descriptive study of female roller derby athletes' beliefs about risk factors for injury in roller derby. BMJ Open Sport Exerc Med 2017;3:e000294. doi:10.1136/bmjsem-2017000294

Accepted 12 September 2017

\section{CrossMark}

Department of Applied Health, Southern Illinois University Edwardsville, Edwardsville, Illinois, USA

Correspondence to Dr Michelle L Cathorall; mcathor@siue.edu

\section{ABSTRACT \\ Objectives To examine skaters' beliefs about risk factors related to roller derby injuries.}

Design Qualitative study based on semistructured focus group discussions with female roller derby skaters.

Methods Skaters with varying levels of experience and injury history were invited to participate in focus group discussions. Semistructured focus group discussions were based on the question 'What factors do you believe contribute to roller derby injuries?'. Analysis of the data were organised into themes, summarised and interpreted, separated based on being an intrinsic or extrinsic factor and then reorganised by frequency of citation. Focus groups continued until no new themes were added to the data.

Results The factors discussed by derby skaters were divided into intrinsic and extrinsic factors. The most common intrinsic factors cited were behavioural and psychological, 'knowing one's body' and internal pressure related to 'not letting team down'. The most cited extrinsic factors were 'unmatched skill level' and 'poor quality' equipment.

Conclusions Female roller derby skaters contribute the greatest injury risk to new skaters not knowing their body and experienced skaters not considering new skaters skill levels. Knowing the factors that skaters perceive as increasing injury risk may contribute to interventions to decrease the frequency and severity of injuries. New findings include behavioural, psychological and social factors related to injury risk. Important considerations for healthcare providers treating roller derby skaters include the psychological and social factors that affect injury.

\section{INTRODUCTION}

Roller derby is a full contact sport where skaters score by skating around a track passing the opposing skaters. To stop the other team from scoring, skaters must physically block their opponents, thus physical contact between skaters is necessary. The sport is a combination of speed, agility and stamina, similar to sports such as soccer, football and hockey. Thus, the benefits of roller derby as a form of physical activity may be similar to other sports, such as decreased chronic disease risk and

\section{What are the new findings?}

- Players cited several psychological, behavioural social and environmental factors related to injuries.

- More experienced players with a history of a roller derby injury cited the internal pressure to return to play too quickly after an injury and being distracted or fatigued as causes of injury. Players regardless of experience indicated that poor quality equipment was related to injury.

How might it impact clinical practice in the near future?

- It is important for healthcare providers to consider the psychological and social factors related to roller derby injuries when developing treatment plans for injured skaters to help ensure compliance with the therapeutic regimen.

- Incorporating discussions about quality equipment with skaters seeking care may help reduce future injuries.

improved general health. ${ }^{1}$ Despite the benefits associated with increased physical activity skaters may experience playing roller derby, participating in sports increases the risk of injury. ${ }^{2}$ One criterion for continued participation in sports is safety $^{3}$ and risk reduction. While women's roller derby has had a resurgence in popularity since 2006, with over 30000 skaters from 79 countries, ${ }^{4}$ little is known or understood about injuries or the risk factors for them.

In a sample of 14000 skaters, 53\% reported sustaining at least one injury requiring medical attention in the previous 12 months. ${ }^{5}$ Some factors inherent to the sport including the surfaces the sport is played on, speed, quick stops and changes in direction, the instability of being on skates and physical contact between players 
may increase the risk of injury. As with other sports, roller derby's organising bodies have safety guidelines and injury protocols that must be followed by registered leagues. ${ }^{6}$ The focus of these policies is on facilities, procedures and equipment. Additional policies to increase player safety include ensuring that all players can demonstrate the minimum skills required for safe roller derby play. ${ }^{7}$ While these policies address important factors related to the aetiology of injuries, they do not address contextual or behavioural injury risk factors.

Most roller derby injuries are acute traumatic injuries related to a blow or force. To our knowledge, no literature is available that examines how roller derby skaters' think about the risk factors for their sport. There may be behavioural and contextual factors that contribute to injuries that are not yet understood. Understanding roller derby skaters' beliefs has the potential to increase the effectiveness of prevention programme by addressing them during programme development and implementation. This is critical as research indicates that in order for prevention measures to be institutionalised, we must change behaviour and the end users must understand and accept the determining and influencing factors for sports safety behaviours. ${ }^{28}$ Although roller derby popularity continues to grow and there are a concerning number of injuries sustained, to date, there are no published studies that have examined factors related to injury risk in this sport. To this end, we conducted a qualitative study to compile and explore perceived injury risk factors in roller derby from the skaters' perspective.

\section{METHODS}

Since this is the first study to examine this phenomenon, we conducted a phenomenological qualitative study using semistructured focus group discussions. Eight Women's Flat Track Derby Association (WFTDA) leagues were initially contacted via their league Facebook page or email and asked to send the information to their members. Interested members were asked to contact the investigators. Focus groups were scheduled with the skaters in the order that they contacted us, representing five leagues. Leagues contacted for this study were located within a 3-hour driving radius from the investigators' institution. Inclusion criteria were being an active registered skater with a WFTDA league and being 18 years of age or older. Inclusion was restricted to those leagues in WFTDA because the association has clear safety policies and minimum skills requirements for all active skaters. The remaining inclusion criteria were minimal to ensure that a wide range of experience skating and derby injuries were included. Sample size was determined when data saturation was reached. The institutional review board at Southern Illinois University Edwardsville approved this study. All participants read the consent form and were given the opportunity to ask questions prior to signing the form. To protect participants' rights they could refuse to answer any question they were uncomfortable with. Participants were able to choose a pseudonym for the audio recording to protect their anonymity.

To understand the factors related to roller derby injuries, we conducted focus groups to compile skaters' beliefs and opinions. Focus group discussions were conducted between July 2015 and March 2016 in public places, which were chosen by the participants. The same interviewers conducted all the focus group discussions. The interviewers were trained to pause and not fill gaps in the conversation to allow participants to freely talk about their experiences and beliefs. Prior to scheduling, focus groups participants were screened for self-reported injury status. A roller derby injury was defined as one that occurred while playing or practising roller derby with their league, required medical attention in the last 12 months and resulted in missing 10 or more days of roller derby. This screening was done to ensure that skaters with prior injury were interviewed together and those with no history of injury were interviewed together. As an incentive, participants received a gift card for a local skate shop at the end focus group. Focus groups were scheduled so skaters from the same league could participate together. Participants were asked to complete a brief questionnaire that included demographic and roller derby history questions. All participants answered the same questionnaire. Focus groups consisted of up to five skaters. All focus groups began with the question 'Why did you start playing roller derby?' to get them comfortable talking with us. Skaters were then asked 'What factors do you believe contribute to roller derby injuries?' Focus groups were continued until no new information emerged from the discussions. The discussions lasted $50 \mathrm{~min}$ on average. Participant responses were audio recorded directly to a password-protected laptop. The recordings were transcribed verbatim to a text document by one of the investigators. The principal investigator checked the accuracy of the transcribed material by comparing at least four $5 \mathrm{~min}$ sections of each transcript to the recorded material.

Descriptive analysis of participant characteristics was conducted. Analysis of the transcripts was performed in three steps. In step 1, data were initially coded into themes to describe what the participants were describing based on the participants' own words or phrases. In step 2 , the data were condensing into focused codes or factors. In this step, the thematic units were interpreted by the investigators and summarised in one or two words. Both investigators agreed on initial codes and focused codes created during data analysis. The final step was to organise the factors into intrinsic or extrinsic categories by frequency of citation. Intrinsic factors were defined as those things that were internal to the participant, such as age, roller derby history, injury status, behaviour and skill level. Extrinsic factors included those things 


\begin{tabular}{ll}
\hline \multicolumn{2}{l}{ Table 1 Participant characteristics $(\mathrm{n}=19)$} \\
\hline Characteristic & Value \\
\hline Age, years* & $29.42 \pm 6.552$ \\
\hline Years playing roller derby* & $2.75 \pm 2.9$ \\
\hline Hours of roller derby per week* & $1.882 \pm 1.1315$ \\
\hline Hours of other physical activity per & \\
week, $n$ (\%) & \\
\hline None & $1(5)$ \\
\hline Once a week & $4(21)$ \\
\hline Twice a week & $2(11)$ \\
\hline Three times a week & $4(21)$ \\
\hline Four times a week & $4(21)$ \\
\hline Five times a week & $4(21)$ \\
\hline Roller derby injury, $n(\%)$ & \\
\hline Yes & $14(73.7)$ \\
\hline No & $5(26.3)$ \\
\hline
\end{tabular}

*Values are mean \pm SD.

that are external to the skater such as equipment, practice space, organisational culture and other skaters' skill level.

\section{RESULTS}

Nineteen skaters, from five leagues, between the ages of 23 and 36 years participated (mean age 29.4 years). Skaters had an average of 2.75 years of roller derby experience and almost three-quarters (74\%) had experienced a roller derby injury. After the fifth focus group, no new information emerged, confirming saturation. The characteristics of participants are described in table 1. Based on the data analysis, intrinsic factors were separated into seven categories: derby culture buy-in, behaviour, psychological, fatigue, lack of knowledge, physical ability and skill level. Extrinsic factors were divided into seven categories: derby culture, training, competitor-related factors, equipment, aspects of the sport, facility and other.

For intrinsic factors (table 2), the behavioural category was the most often cited by participants. The most common phrases used were 'pushing too hard' and 'knowing one's body'. Participants discussed pushing themselves too hard to try advanced skills and to scrimmage before they were ready. As one skater noted 'I tried to do more advanced things quicker than I should have and it ended up with me injuring myself'. Skaters mentioned that even when they thought they should not skate they often felt the need to push themselves to get better. As one skater discussed the internal pressure to get back to skating is so strong that it can keep injuries from healing '... it didn't fully
Table 2 Intrinsic factors reported by participants

\begin{tabular}{|c|c|}
\hline Category/factor & Number of citations \\
\hline \multicolumn{2}{|l|}{ Derby culture buy-in } \\
\hline Pride in old gear & 4 \\
\hline Pride in injury & 3 \\
\hline \multicolumn{2}{|l|}{ Behaviour } \\
\hline Internal pressure & 11 \\
\hline Knowing one's body & 14 \\
\hline Not cross-training & 3 \\
\hline Pushing self too hard & 16 \\
\hline Overtraining & 3 \\
\hline Not seeking medical attention & 2 \\
\hline \multicolumn{2}{|l|}{ Psychological } \\
\hline Head not in the game & 8 \\
\hline Attitude & 3 \\
\hline Competitiveness & 1 \\
\hline Fatigue & 7 \\
\hline \multicolumn{2}{|l|}{ Lack of knowledge } \\
\hline Need for cross-training & 3 \\
\hline Equipment & 2 \\
\hline \multicolumn{2}{|l|}{ Physical ability } \\
\hline Clumsy & 3 \\
\hline Muscles are not developed & 2 \\
\hline Weak & 3 \\
\hline \multicolumn{2}{|l|}{ Skill level } \\
\hline Lack of experience & 5 \\
\hline
\end{tabular}

heal until earlier this year...because I just kept going back out there, because that is what we do...' The idea of pushing oneself seems to be related to internal pressure to not let the team down. One skater discussed breaking her ankle during a scrimmage and the idea that she would not be able to play any more, '...I knew what it meant, and I felt like I had just disappointed my team'. Experienced skaters noted that new skaters or skaters who did not have a history of playing sports did not know their body enough to know when it was sore versus injured. Participants admitted to walking around with serious injuries such as a broken foot and a concussion for days before realising that they may be injured.

The second most often cited intrinsic category was psychological factors with the most common factor cited being 'head not in the game'. Skaters discussed the mental aspects of the game, not knowing where you are or other skaters are on the track as risk factors for injury. Skaters mentioned how having a bad day at 
Table 3 Extrinsic factors reported by participants

\begin{tabular}{ll}
\hline Category/factor & Number of citations \\
\hline Derby culture & \\
Tough image & 2 \\
External pressure & 6
\end{tabular}

Training

\begin{tabular}{|ll}
\hline Knowing basic derby skills & 2 \\
\hline Injury identification & 3 \\
\hline Lack of team cross-training & 1 \\
\hline Competitor-related factors & \\
\hline Unmatched skill level & 9 \\
\hline Equipment & \\
\hline Poor quality & 7 \\
\hline Wrong size & 3 \\
\hline Maintenance & 1 \\
\hline Aspects of the sport & 1 \\
\hline Getting hit/hitting people & 2 \\
\hline On four wheels & 3 \\
\hline Quick movements & 1 \\
\hline Facility & 3 \\
\hline Track surface & \\
\hline Temperature & \\
\hline Other & \\
\hline Freak accident & \\
\hline
\end{tabular}

work and going to practice can cause injuries if someone is distracted and not focused on proper form and what is happening on the track. Skaters with a history of a serious roller derby injury reported feeling 'fatigue' when they were injured. Whereas this was not a factor discussed by skaters without a history of injury.

For the extrinsic factors (table 3), the majority of skaters believed that competitor-related factors and equipment issues were the main causes of injury in roller derby. The most often cited extrinsic factor that cause injuries according to participants was 'unmatched skill level'. The second most cited factor was 'poor quality' equipment. Skaters discuss the difference between skating with more players of higher skill levels as the biggest extrinsic risk factor for injury. Skaters mentioned getting hit by players at a higher skill level than them and being injured, whereas they felt that the same risk is not there when they skate with players at the same level. As one skater explained, '...we don't practice together, they don't know our skill level...they don't know what is ok'. More experienced skaters spoke of new skaters purchasing poor quality, but inexpensive gear as the second leading risk factor for injuries. Quality roller derby pads are expensive and as one skater pointed out, 'I think a lot of newer skaters, their gear choice is dictated by their budget'. As one skater explained, 'I see a lot of new girls in cheap knee pads and that worries me. Like a lot of girls will go to [a sporting good store] and buy a roller blade pack. It's 20 bucks for knee, elbow and wrist pads. We fall a lot harder than that though'. Often new skaters will purchase inexpensive but inferior equipment while they decide if they want to continue with roller derby and invest in roller derby equipment.

\section{DISCUSSION}

This is the first study, to our knowledge, to examine skaters' views on roller derby injury risk factors. The sample consisted of 19 female roller derby skaters, who were on average 29 years old with an average of 2.75 years' experience playing roller derby. Almost threequarters of the skaters had previously sustained a roller derby-related injury. The most often cited intrinsic factors mentioned by participants were pushing too hard, knowing one's body and internal pressure. The most common extrinsic factors cited were unmatched skill level and poor quality equipment.

While these findings provide an outline of skaters' beliefs about injury risk factors, not the full scope of risk factors, this study provides an insight into the implementation context from the perspective of the end user. Considering the end user is critical to the successful implementation and compliance with any injury prevention intervention. One area where there is a dearth of research in injury prevention is in understanding the contextual variables that affect implementation of safety and injury prevention interventions. These topics have not yet been examined for roller derby. Understanding skaters' perceptions of what they believe increases the risk of injury for roller derby players is key to developing interventions that are acceptable to players, coaches and organisations. ${ }^{9}$ The results of this study correspond to key components of stage 5 of the Translating Research into Injury Prevention Practice framework. ${ }^{9}$ This model expands the previous sports injury prevention models by building the contextual relationships into the model. Stage 5 posits that understanding the perceptions of the players, how likely they believe it is that they will be injured playing and the safety and injury culture of a particular sport is necessary to for evaluating implementation and ultimately effectiveness of the intervention. ${ }^{9}$ This preliminary list could be used in understanding the safety and injury culture of roller derby.

The results should be viewed with caution as the size and diversity of our sample and the study design prevent generalisability of the results. First, we sampled 19 skaters out of approximately 30000 worldwide. ${ }^{4}$ Second, the sample is drawn from the midwest 
roller derby skaters. As beliefs and knowledge are influenced by culture, findings from this study cannot be generalised to roller derby skaters in other parts of the country or world. Third, study design does not allow for verification of the findings, thus we do not know that these perceived risk factors are true risk factors for injury in roller derby. More work is needed to verify the findings with a larger more diverse sample of skaters.

In conclusion, women's roller derby is a growing sport, and the risk factors for injury are poorly understood. This preliminary investigation has identified important themes related to skaters' perceived injury risk factors in roller derby, contributing to the emerging body of knowledge on roller derby injuries. The skaters' perspective may help identify potential, context-relevant intervention strategies to reduce injury risk. To understand what causes roller derby injuries, it is important to understand the aetiology of them and to determine environmental and behavioural factors that contribute to injury events. To increase effectiveness for roller derby injury prevention programme, it is important to incorporate pre-existing beliefs of skaters about intrinsic and extrinsic factors related to roller derby injuries. Prevention programmes should incorporate equipment, training methods and monitoring strategies for skaters and coaches. An important environmental risk factor is derby culture and the tough image that is associated with it. Changing the perception and acceptance of this may help reduce the pressure felt by skaters to uphold the image, taking unnecessary risks that increase injuries. Findings may also help health professionals treat injured roller derby skaters by having a better understanding of skaters' beliefs related to roller derby injuries and the pressure felt to return to the play.

Acknowledgements The Southern Illinois University Edwardsville's Undergraduate Research and Creative Activities Program provided financial assistance to support MP as an undergraduate research assistant for one semester on this study.
Contributors MLC initiated the study, making significant contributions to the design with input from MP. Both authors participated equally in the data collection process. MP conduced the initial analysis/interpretation with input and guidance from MLC. Both authors agreed on the final analysis. MP wrote the initial draft of the manuscript with significant revisions by MLC for the final version.

Competing interests None declared.

Patient consent Obtained.

Ethics approval The Institutional Review Board at Southern Illinois University Edwardsville approved the study procedures to ensure human rights and ethical guidelines were followed. The approval number is IRB \#15-0521-2.

Provenance and peer review Not commissioned; internally peer reviewed.

Data sharing statement The manuscript describing the unpublished epidemiological data mentioned in this study is currently being written. The data manuscript will be submitted for publication in the next month.

Open Access This is an Open Access article distributed in accordance with the Creative Commons Attribution Non Commercial (CC BY-NC 4.0) license, which permits others to distribute, remix, adapt, build upon this work noncommercially, and license their derivative works on different terms, provided the original work is properly cited and the use is non-commercial. See: http:// creativecommons.org/licenses/by-nc/4.0/

(C) Article author(s) (or their employer(s) unless otherwise stated in the text of the article) 2017. All rights reserved. No commercial use is permitted unless otherwise expressly granted.

\section{REFERENCES}

1. Pedersen BK, Saltin B. Evidence for prescribing exercise as therapy in chronic disease. Scand J Med Sci Sports 2006;16 Suppl 1:3-63.

2. Verhagen EA, van Mechelen W. Sport for all, injury prevention for all. Br J Sports Med 2010;44:158.

3. van Wilgen $\mathrm{CP}$, Verhagen $\mathrm{EA}$. A qualitative study on overuse injuries: the beliefs of athletes and coaches. J Sci Med Sport 2012;15:116-21.

4. Women's Flat Track Derby Association (WFTDA) website. WFTDA releases demographic survey and results of 2015 census. https:// wftda.org/news/wftda-releases-demographic-survey-and-results-of2015-census 2009-2016 (accessed 18 July 2017).

5. Cathorall ML, Peachey A, . The epidemiology of injuries among female roller derby skaters poster. The 2016 Society for Public Health Education Conference; March 2016, Charlotte, NC.http://www.siue. edu/ mcathor/cathorall-peachey-_the.pdfaccessed 17 Jul 2017

6. Women's Flat Track Derby Association (WFTDA) risk management guidelines website. $2017 \mathrm{https} / / /$ static.wftda.com/resources/ insurance/wftda-risk-management-guidelines.pdf (accessed $31 \mathrm{Jul}$ 2017).

7. Women's Flat Track Derby Association (WFTDA) training website. https://wftda.com/the-game/training/ 2009-2017 (accessed 31 July 2017).

8. Verhagen EA, van Stralen MM, van Mechelen W. Behaviour, the key factor for sports injury prevention. Sports Med 2010;40:899-906.

9. Finch C. A new framework for research leading to sports injury prevention. J Sci Med Sport 2006;9:3-9. 